TRANSACTIONS OF THE

AMERICAN MATHEMATICAL SOCIETY

Volume 287, Number 2, February 1985

\title{
ON THE GAUSS-BONNET THEOREM FOR COMPLETE MANIFOLDS
}

\author{
$\mathrm{BY}$
}

STEVEN ROSENBERG

\begin{abstract}
For a manifold diffeomorphic to the interior of a compact manifold with boundary, several classes of complete metrics are given for which the Gauss-Bonnet Theorem is valid.
\end{abstract}

Introduction. For a compact oriented Riemannian manifold $M$, the GaussBonnet Theorem states that $\chi(M)=\int_{M} E(g)$, where $E(g)$ is the Euler form for the metric $g$. For noncompact manifolds the theorem is known to hold on finitely connected, finite volume Riemann surfaces and for the invariant metric on quotients of symmetric spaces by discrete, torsion-free, arithmetic subgroups. The proofs are due to $[\mathbf{H u}]$ and Harder $[\mathbf{H a}]$ respectively (see also $[\mathbf{G r}$, p. $84 ; \mathbf{C G}]$ ).

A manifold in either of these classes is diffeomorphic to the interior of a compact manifold with boundary. This is classical for Riemann surfaces, while for arithmetic domains the result follows from the reduction theory of Borel and Harish-Chandra. Elementary Morse theory thus guarantees that there are coordinates on a collar $\partial M \times \mathbf{R}^{+}$of the boundary in which the metric may be written $g=g_{1}(x, t)+d t^{2}$, where $x \in \partial M$ and $t \in \mathbf{R}^{+}$. In [Ro] this form of the metric gives rise to a short proof of Huber's result.

In $\S 1$ we exhibit, through the use of moving frames, several classes of complete metrics for which Gauss-Bonnet is valid. For example, in Theorem 1.9 we show that if $g$ is a warped product metric, $g=f^{2}(t) g_{1}+d t^{2}$ for $g_{1}$ a metric on $\partial M$, then for Gauss-Bonnet it suffices that $f \rightarrow 0$ and $f^{\prime} \rightarrow 0$ as $t \rightarrow \infty$. Since $E(g)$ depends on the second derivatives of $g$, it is surprising at first glance that only first order conditions on $f$ are needed. However, roughly speaking, the warped product structure controls the second order information.

All the metrics in $\S 1$ are more or less modeled on (1.14), Borel's explicit form of the invariant metric for arithmetic quotients of split rank-one symmetric spaces. Even with this explicit form of the metric, Harder's result is not trivial (see Theorem 1.13). The calculation gives a glimpse of the delicate interplay between the algebra and geometry of these spaces.

In $\S 2$ we give some other metrics for which Gauss-Bonnet holds. In particular, we show how to construct many surfaces of positive curvature and infinite volume with Gauss-Bonnet. This contrasts with Huber's result, since in some sense finite volume surfaces must be mostly negatively curved near infinity.

This paper is a revised version of part of the author's thesis. He would like to thank his advisor, Joseph Wolf, for his supervision and continued encouragement.

Received by the editors December 19, 1983 and, in revised form, February 13, 1984.

1980 Mathematics Subject Classification. Primary 53C20; Secondary 53C35.

(C)1985 American Mathematical Society $0002-9947 / 85 \$ 1.00+\$ .25$ per page 
He would also like to thank Harold Donnelly for pointing out an error in the original version.

1. Let $M$ be a complete oriented Riemannian manifold diffeomorphic to the interior of a compact manifold $\bar{M}$ with boundary $\partial \bar{M}=\partial M$. Such a manifold is said to be of finite topological type. Recall that the Euler form $E(g)$ of a metric $g$ is the Pfaffian of the curvature two-form associated to $g$. We wish to find conditions on $g$ which guarantee that $\chi(M)=\int_{M} E(g)$.

As in the compact case, for odd-dimensional $M$ the question is purely topological. The Euler form vanishes pointwise by the formulas in $[\mathbf{C h}]$ or, alternatively, by the fact that the Pfaffian of an odd-dimensional skew-symmetric matrix is zero. Moreover, if $2 \bar{M}$ is the double of $\bar{M}, 0=\chi(2 \bar{M})=2 \chi(\bar{M})-\chi(\partial M)=2 \chi(M)-$ $\chi(\partial M)$. This gives the following result.

LEMMA 1.1. Let $M$ be an odd-dimensional oriented manifold of finite topological type. Then

$$
\chi(M)=\int_{M} E(g) \text { if and only if } \chi(\partial M)=0 .
$$

For example, let $M=\Gamma \backslash G / K$ be the quotient of a split rank one symmetric space $G / K$ by a discrete, torsion-free, arithmetic subgroup $\Gamma$. It follows from reduction theory that each component $\partial M^{i}$ of $\partial M$ is the total space of a fibration over a torus $T_{1}$ with a torus $T_{2}$ as fiber [Bo, Chapter III, §17]. By a standard spectral sequence argument [Sp, p. 481], $\chi\left(\partial M^{1}\right)=\chi\left(T_{1}\right) \chi\left(T_{2}\right)=0$, which verifies Harder's result for this case.

Before going on to even-dimensional manifolds, we need to review Chern's GaussBonnet Theorem for manifolds with boundary $[\mathbf{C h}]$. On any Riemannian manifold, a local orthonormal frame of one-forms $\theta^{i}$ determines the connection one-forms $\omega_{i j}$ by the equations

$$
d \theta^{i}=\sum_{j} \omega_{i j} \wedge \theta^{j} \quad \text { and } \quad \omega_{j i}=-\omega_{i j}
$$

The curvature two-forms are then given by

$$
\Omega_{i j}=d \omega_{i j}-\sum_{k} \omega_{i k} \wedge \omega_{k j}
$$

For an $n$-dimensional Riemannian manifold $M$, let $S M$ denote the $(2 n-1)$-dimensional manifold of unit tangent vectors. To a point $(x, \xi)$ in the fiber of $S M$ over $x \in M$, we associate a frame $\left\{\theta^{1}, \ldots, \theta^{n}\right\}$ of $M$ at $x$ with $\theta^{1}$ dual to $\xi$. From the associated $\omega_{i j}$ and $\Omega_{i j}$ we construct the form

$\Pi(g)=\sum_{0 \leq k<n} c_{k} \cdot \sum_{\alpha} \operatorname{sgn}(\alpha) \Omega_{\alpha(2) \alpha(3)} \wedge \cdots \wedge \Omega_{\alpha(2 k) \alpha(2 k+1)} \wedge \omega_{\alpha(2 k+2) 1} \wedge \cdots \wedge \omega_{\alpha(n) 1}$.

The $c_{k}$ are constants whose values are unimportant to us. The second sum is over all permutations $\alpha$ of $\{2,3, \ldots, n\}$. One checks that $\Pi(g)$ transforms like an $(n-1)$ form on $M$, i.e. $\Pi(g) \in \operatorname{pr}^{*} \Lambda^{n-1}(m)$, the pull-back of the $(n-1)$-forms on $M$ by the projection pr: $S M \rightarrow M$. To be more precise, if $\mu$ is a tangent vector to $S M$ at $(x, \xi)$, then $\mu=\mu_{1}+\mu_{2}$ with $\mu_{1}$ tangent to $M$ and $\mu_{2}$ tangent to the fiber $S_{x}^{n-1}$ at $x$; we have $\Omega_{i j}(\mu)=\Omega_{i j}\left(\mu_{1}\right)$ and similarly for $\omega_{i 1}(\mu)$. 
Now suppose $M$ is incomplete with boundary $\partial M$. Then Chern's theorem is

$$
\chi(M)=\int_{M} E(g)+\int_{\partial M} \rho^{*} \Pi(g) .
$$

Here $\rho$ is the section of $S M$ over $\partial M$ given by the outward unit normal vector. Since $\rho^{*} \Omega_{i j}(\mu)=\Omega_{i j}\left(\mu_{1}\right)$, and similarly for $\omega_{i 1}$, we can just write

$$
\chi(M)=\int_{M} E(g)+\int_{\partial M} \Pi(g) .
$$

From here on, $M$ will be even dimensional and of finite topological type. We will first choose "gradient flow" coordinates which simplify the components of the metric $g$ near $\partial M$. Any Morse function $f$ on $M$ has no critical points in an open neighborhood of $\partial M$, so the hypersurfaces $\{f=$ constant $\}$ are all diffeomorphic in this neighborhood. Let $\left(x_{1}, \ldots, x_{n-1}\right)$ be local coordinates on one fixed hypersurface and let $x_{n}$ be the arclength parameter for the trajectories orthogonal to the hypersurfaces (see [Mi, Chapter I]). Thus the component $g_{i j}$ of $g$ in the coordinates $\left(x_{1}, \ldots, x_{n}\right)$ satisfies $g_{i n}=0,1 \leq i \leq n-1$, and $g_{n n}=1$. Note that since $M$ is complete, the ends of $M$ are covered by such coordinate charts with $x_{n}$ ranging over $(0, \infty)$. Note also that the $x_{n}$ coordinate is the same in overlapping coordinate charts of this type.

For $h \in(0, \infty)$, let $M_{h}$ be all of $M$ except for points $\left(x_{1}, \ldots, x_{n}\right)$ in the ends with $x_{n}>h$. Then

$$
\chi(M)=\chi\left(M_{h}\right)=\int_{M_{h}} E(g)+\int_{\partial M_{h}} \Pi(g) .
$$

Thus $M$ will satisfy Gauss-Bonnet if $\Pi(g)$ goes to zero uniformly on $\partial M_{h}$ as $h$ goes to infinity.

We know that the tangent bundle $T M$ splits in each end as $T M=W \oplus \mathbf{R}$, where $W$ is an integrable distribution. Motivated by the rank-one arithmetic domain example, we now assume that $W$ splits as

$$
W=W_{2} \oplus W_{3} \oplus \cdots \oplus W_{k}, \quad k \geq 2, \quad \text { with }\left[W_{i}, W_{j}\right]=0 \quad \text { if } i \neq j .
$$

We also assume that, with respect to this splitting, $g$ has the form

$$
g=f_{2}^{2}\left(x_{n}\right) g_{2} \oplus \cdots \oplus f_{k}^{2}\left(x_{n}\right) g_{k}^{2}+d x_{n}^{2}
$$

with $g_{i}$ a metric on $W_{i}$ and $f_{i}$ chosen positive. If $\partial M_{0}$ is a product manifold $N_{1} \times \cdots \times N_{k}$ and if $W_{i}=T N_{i}$, then (1.5) is called a (multiply) warped product metric. In general, however, we will not even assume that the $W_{i}$ are integrable. As explained below (Theorem 1.13), the motivation for (1.5) is also the invariant metric on rank-one arithmetic domains.

We now want to estimate $\Pi(g)$ for the metric (1.5) to see what conditions on the $f_{i}$ will ensure Gauss-Bonnet. We first select a local orthonormal frame at $\partial M_{1},\left\{\theta^{1}, \theta^{2 \alpha}, \ldots, \theta^{k \alpha}\right\}$, with $\theta^{1}=d x_{n}$ and $\left\{\theta^{j \alpha}\right\}, \alpha=1, \ldots, \operatorname{dim} W_{j}$, a local orthonormal frame for $W_{j}$. A frame at $\partial M_{h}$ is then given by

$$
\left\{d x_{n}, \frac{f_{2}(h)}{f_{2}(1)} \theta^{2 \alpha}, \ldots, \frac{f_{k}(h)}{f_{k}(1)} \theta^{k \alpha}\right\},
$$

which for general $h$ will be denoted by $\left\{\bar{\theta}^{1}, \bar{\theta}^{2 \alpha}, \ldots, \bar{\theta}^{k \alpha}\right\}$. We use the indices $\mu, \nu, \rho$ to denote any index in $\{1,2 \alpha, \ldots, k \alpha\}$. 
Now

$$
d \theta^{\mu}=\frac{1}{2} \sum_{\nu, \rho} b_{\nu \rho}^{\mu} \theta^{\nu} \wedge \theta^{\rho} \quad \text { with } b_{\nu \rho}^{\mu}=-b_{\rho \nu}^{\mu}
$$

and

$$
d \bar{\theta}=\frac{1}{2} \sum_{\nu, \rho} \bar{b}_{\nu \rho}^{\mu} \bar{\theta}^{\nu} \wedge \bar{\theta}^{\rho}
$$

We claim that

$$
\begin{array}{ll}
\bar{b}_{\nu \rho}^{1}=0, & \\
\bar{b}_{l \beta, j \gamma}^{j \alpha}=0 & \text { if } j \neq l, \\
\bar{b}_{l \beta, r \gamma}^{j \alpha}=0 & \text { if } l \neq r, \\
\bar{b}_{1, j \beta}^{j \alpha}=0 & \text { if } \alpha \neq \beta, \\
\bar{b}_{1, l \beta}^{j \alpha}=0 & \text { if } j \neq l .
\end{array}
$$

Here $j, l, r$ range from 2 to $k$, and $\alpha, \beta, \gamma$ range from 1 to the dimension of the appropriate $W_{i}$. The first question follows from $d \bar{\theta}^{1}=0$. For the next equation, set $\left\{\bar{X}_{1}, \bar{X}_{2 \alpha}, \ldots, \bar{X}_{k \alpha}\right\}$ to be the frame of tangent vectors dual to the $\bar{\theta}$ 's. Then

$$
\begin{aligned}
\bar{b}_{l \beta, j \gamma}^{j \alpha} & =d \bar{\theta}^{j \alpha}\left(\bar{X}_{l \beta}, \bar{X}_{j \gamma}\right) \\
& =\bar{X}_{l \beta}\left(\bar{\theta}^{j \alpha}\left(\bar{X}_{j \gamma}\right)\right)-\bar{X}_{j \gamma}\left(\bar{\theta}^{j \alpha}\left(\bar{X}_{l \beta}\right)\right)-\bar{\theta}^{j \alpha}\left(\left[\bar{X}_{l \beta}, \bar{X}_{j \gamma}\right]\right) \\
& =0
\end{aligned}
$$

if $l \neq j$. The third equation follows similarly. Finally, an easy calculation shows that $\left[\bar{X}_{1}, \bar{X}_{j \alpha}\right]=\left[\partial / \partial x_{n}, \bar{X}_{j \alpha}\right]$ is a multiple of $\bar{X}_{j \alpha}$ so, as above, the last two equations follow.

The nonvanishing $\bar{b}$ 's can be determined by

$$
\begin{aligned}
d \bar{\theta}^{j \alpha}= & d\left(\frac{f_{j}(h)}{f_{j}(1)} \theta^{j \alpha}\right)=\frac{1}{f_{j}(1)}\left[\frac{\partial f_{j}}{\partial x_{n}} \theta^{1} \wedge \theta^{j \alpha}+f_{j} d \theta^{j \alpha}\right] \\
= & \frac{1}{f_{j}} \frac{\partial f_{j}}{\partial x_{n}} \bar{\theta}^{1} \wedge \bar{\theta}^{j \alpha}+\frac{f_{j}}{f_{j}(1)}\left(\frac{1}{2} \sum_{\nu, \rho} b_{\nu, \rho}^{j \alpha} \theta^{\nu} \wedge \theta^{\rho}\right) \\
\bullet= & \left(\frac{1}{f_{j}} \frac{\partial f_{j}}{\partial x_{n}}+b_{1, j \alpha}^{j \alpha}\right) \bar{\theta}^{1} \wedge \bar{\theta}^{j \alpha} \\
& +\frac{f_{j}}{f_{j}(1)}\left(\frac{1}{2} \sum_{\nu, \rho}^{\prime} b_{\nu, \rho}^{j \alpha} \frac{f_{\nu}(1) f_{\rho}(1)}{f_{\nu} f_{\rho}} \bar{\theta}^{\nu} \wedge \bar{\theta}^{\rho}\right)
\end{aligned}
$$

$\left(\sum^{\prime}\right.$ denotes the sum over all pairs $(\nu, \rho)$ not equal to $(1, j \alpha)$ or $\left.(j \alpha, 1)\right)$. In other words,

$$
\bar{b}_{1, j \alpha}^{j \alpha}=b_{1, j \alpha}^{j \alpha}+\frac{1}{f_{j}} \frac{\partial f_{j}}{\partial x_{n}}, \quad \bar{b}_{l \beta, l \gamma}^{j \alpha}=\frac{f_{j} \cdot f_{l}^{2}(1)}{f_{j}(1) f_{l}^{2}} \cdot b_{l \beta, l \gamma}^{j \alpha} .
$$

For any metric,

$$
d \bar{\theta}^{\mu}=\frac{1}{2} \sum_{\nu, \rho} \bar{b}_{\nu \rho}^{\mu} \bar{\theta}^{\nu} \wedge \bar{\theta}^{\rho} \quad \text { implies } \quad \bar{\omega}_{\mu \nu}=\sum_{\rho} \bar{a}_{\nu \rho}^{\mu} \bar{\theta}^{\rho}
$$


with

$$
\bar{a}_{\nu \rho}^{\mu}=\frac{1}{2}\left(\bar{b}_{\nu \rho}^{\mu}+\bar{b}_{\rho \mu}^{\nu}-\bar{b}_{\mu \nu}^{\rho}\right) .
$$

A straightforward calculation from (1.7) gives

$$
\bar{\omega}_{1, j \alpha}=2\left(b_{1, j \alpha}^{j \alpha}-\frac{1}{f_{j}} \frac{\partial f_{j}}{\partial x_{n}}\right) f_{j} \theta^{j \alpha} .
$$

If (1.5) represents a warped product metric, the calculation of $\Pi$ becomes easier. In this case, $d \bar{\theta}^{j \alpha}=d_{j} \bar{\theta}^{j \alpha}$, where $d_{j}$ is the exterior derivative for the submanifold $N_{j}$. Thus

$$
\begin{aligned}
d \bar{\theta}^{j \alpha} & =\frac{f_{j}}{f_{j}(1)} d_{j} \theta^{j \alpha}+\frac{1}{f_{j}(1)} \frac{\partial f_{j}}{\partial x_{n}} \theta^{1} \wedge \theta^{j \alpha} \\
& =\frac{f_{j}}{f_{j}(1)} \sum_{\beta} \omega_{j \alpha, j \beta} \wedge \theta^{j \beta}+\frac{1}{f_{j}(1)} \frac{\partial f_{j}}{\partial x_{n}} \theta^{1} \wedge \theta^{j \alpha} \\
& =\sum_{\beta} \omega_{j \alpha, j \beta} \wedge \bar{\theta}^{j \beta}+\frac{1}{f_{j}(1)} \frac{\partial f_{j}}{\partial x_{n}} \bar{\theta}^{1} \wedge \bar{\theta}^{j \alpha}
\end{aligned}
$$

Here $\omega_{j \alpha, j \beta}$ are the connection one-forms for $N_{j}$. Thus $\bar{\omega}_{j \alpha, j \beta}=\omega_{j \alpha, j \beta}$ for $\alpha \neq \beta$ (and trivially for $\alpha=\beta$ ), and $\bar{\omega}_{j \alpha, l \beta}=0$ if $j \neq l$. Similarly, $\bar{\Omega}_{\mu, \nu}=d \bar{\omega}_{\mu \nu}-$ $\sum \bar{\omega}_{\mu \rho} \wedge \bar{\omega}_{\rho \nu}$ is either zero or equal to $\Omega_{\mu \nu}$ (for $\mu, \nu \neq 1$ ). Thus every term in (1.2), except $\bar{\omega}_{j \alpha, 1}$ terms, is bounded. From (1.8), we can now construct many examples of complete manifolds satisfying Gauss-Bonnet.

THEOREM 1.9. Let $M$ be a complete oriented even-dimensional manifold of finite topological type. Assume that in an open neighborhood of each component of the boundary, $M$ splits as a product manifold $M=N_{2} \times \cdots \times N_{k} \times \mathbf{R}$ with metric $f_{2}^{2}(h) g_{2} \oplus \cdots \oplus f_{k}^{2}(h) g_{k} \oplus d h^{2}$, where $g_{j}$ is a metric on $N_{j}$. If $f_{j}(h) \rightarrow 0$ and $f_{j}^{\prime}(h) \rightarrow 0$ as $h \rightarrow \infty$, then $\chi(M)=\int_{M} E(g)$. In particular, any even-dimensional oriented manifold of finite topological type admits complete warped product metrics satisfying Gauss-Bonnet.

The last statement follows by setting $N_{2}=\partial M$. Notice that we only have to impose first order conditions on $f_{j}$ because the warped product structure controls the second order $\bar{\Omega}_{\mu \nu}$.

If the distributions $W_{i}$ are not integrable, further computations are needed. For example,

$$
\begin{aligned}
\bar{\omega}_{j \alpha, j \beta}= & \sum_{\mu} \bar{a}_{j \beta, \mu}^{j \alpha} \bar{\theta}^{\mu} \\
= & \bar{b}_{j \beta, j \alpha}^{j \alpha} \bar{\theta}^{j \alpha}+\bar{b}_{j \alpha, j \beta}^{j \beta} \bar{\theta}^{j \beta} \\
& +\frac{1}{2} \sum_{\gamma \neq \alpha, \beta}\left(\bar{b}_{j \beta, j \gamma}^{j \alpha}+\bar{b}_{j \gamma, j \alpha}^{j \beta}-\bar{b}_{j \gamma, j \beta}^{j \gamma}\right) \bar{\theta}^{j \beta}
\end{aligned}
$$

by (1.6) and the definition of $\bar{a}$. By (1.7) the last expression is unchanged if the bars are deleted, so

$$
\bar{\omega}_{j \alpha, j \beta}=\omega_{j \alpha, j \beta}
$$


Similarly, for $j \neq l$,

$$
\begin{aligned}
\bar{\omega}_{j \alpha, l \beta}= & \frac{1}{2} \sum_{\alpha \neq \gamma} \bar{b}_{j \alpha, j \gamma}^{l \beta} \bar{\theta}^{j \gamma}+\frac{1}{2} \sum_{\beta \neq \gamma} \bar{b}_{l \beta, l \gamma}^{j \alpha} \bar{\theta}^{l \gamma} \\
= & \frac{1}{2} \sum_{\alpha \neq \gamma} \frac{f_{l} \cdot f_{j}(1)}{f_{l}(1) \cdot f_{j}} b_{j \alpha, j \gamma}^{l \beta} \theta^{j \gamma} \\
& +\frac{1}{2} \sum_{\beta \neq \gamma} \frac{f_{j} \cdot f_{l}(1)}{f_{j}(1) \cdot f_{l}} b_{l \beta, l \gamma}^{j \alpha} \theta^{l \gamma} .
\end{aligned}
$$

As before, we want to keep $\bar{\Omega}_{\mu \nu}$ bounded and to have $\bar{\omega}_{1, \rho}$ decay. Now

$$
\begin{aligned}
\bar{\Omega}_{j \alpha, j \beta}= & d \bar{\omega}_{j \alpha, j \beta}-\sum_{\gamma} \bar{\omega}_{j \alpha, j \gamma} \wedge \bar{\omega}_{j \gamma, j \beta} \\
& -\sum_{\substack{l, \gamma \\
l \neq j}} \bar{\omega}_{j \alpha, l \gamma} \wedge \bar{\omega}_{l \gamma, j \beta} .
\end{aligned}
$$

The first two terms on the right are bounded by (1.10). By (1.11), the third term will be bounded if the $f_{j}$ are mutually bounded, i.e. if there exist constants $C_{i j}$, $\tilde{C}_{i j}$ such that for $h \in[1, \infty)$,

$$
\left|f_{j}(h) / f_{i}(h)\right|<C_{i j} \quad \text { and } \quad\left|f_{i}(h) / f_{j}(h)\right|<\tilde{C}_{i j}, \quad i, j=2, \ldots, k .
$$

For $\bar{\Omega}_{j \alpha, j \beta}$, with $j \neq l$, we still have to keep $d \bar{\omega}_{j \alpha, l \beta}$, bounded. This will follow from $(1.11)$ if the derivatives $\left(f_{j} / f_{i}\right)^{\prime}$ and $\left(f_{i} / f_{j}\right)^{\prime}$ are bounded.

THEOREM 1.13. Let $M$ be a compact oriented even-dimensional manifold of finite topological type. Assume that in an open neighborhood of each component of the boundary, $T M=W_{2} \oplus \cdots \oplus W_{k} \oplus \mathbf{R}$ and the metric is of the form $f_{2}^{2}(h) g_{2} \oplus \cdots \oplus$ $f_{k}^{2}(h) g_{k} \oplus d h^{2}$, with $g_{i}$ a metric on $W_{i}$. If $f_{i}(h) \rightarrow 0$ and $f_{i}^{\prime}(h) \rightarrow 0$ as $h \rightarrow \infty$, and if $f_{j} f_{i}^{-1}$ and $\left(f_{j} f_{i}^{-1}\right)^{\prime}$ are bounded for all $h(i, j=2, \ldots, k)$, then $\chi(M)=\int_{M} E(g)$.

We now consider in detail the case of $M=\Gamma \backslash G / K$, an arithmetic quotient of an even-dimensional split rank-one symmetric space. By the remarks after Lemma 1.1, we know that $T M=W_{1} \oplus W_{2} \oplus \mathbf{R}$ over open sets $V \times \mathbf{R}$ whenever the fibration is trivial over $V \subset \partial M$. Here $W_{i}$ is the tangent space to the torus $T_{i}$. Unfortunately, the $G$-invariant metric does not conform to this splitting. More precisely, as explained in [Do] each end of $M$ can be written as $N \times \mathbf{R}$, where $N$ is at most two-step nilpotent. The Lie algebra of $N$ splits into a direct sum $\mathfrak{n}=V_{2} \oplus V_{3}$ of root spaces with $V_{3}=Z(\mathfrak{n})$, the center of $\mathfrak{n}$. The invariant metric at the identity in $N$ is

$$
g=e^{-2 h} g_{2}+e^{-4 h} g_{3}+d h^{2}
$$

with $g_{2}$ a metric on $V_{2}$ and $g_{3}$ a metric on $V_{3}$. Since $[\mathfrak{n}, \mathfrak{n}] \subset Z(\mathfrak{n})$, the $G$-invariant distribution given by $V_{2}$ is not integrable, so Theorem 1.9 cannot be applied in general. (However, for quotients of hyperbolic space $G / K=\mathrm{SO}(n, 1) / \mathrm{SO}(n)$, we have $V_{2}=\mathfrak{n}$, so the theorem applies to give Gauss-Bonnet.)

Although Theorem 1.13 was designed to cover the nonintegrable case, the metric (1.14) does not satisfy the decay hypotheses. However, a closer look at $\mathfrak{n}$ allows us 
to reprove Harder's result. That is, we have

$$
\begin{array}{lll}
{\left[V_{2}, V_{2}\right] \subset V_{3}} & \text { implies } & \bar{b}_{2 \beta, 2 \gamma}^{2 \alpha}=0, \\
{\left[V_{2}, V_{3}\right]=0} & \text { implies } & \bar{b}_{2 \beta, 3 \gamma}^{3 \alpha}=0, \\
{\left[V_{3}, V_{3}\right]=0} & \text { implies } & \bar{b}_{3 \beta, 3 \gamma}^{2 \alpha}=\bar{b}_{3 \beta, 3 \gamma}^{3 \alpha}=0 .
\end{array}
$$

For example,

$$
\bar{b}_{2 \beta, 2 \gamma}^{2 \alpha}=\bar{X}_{2 \beta}\left(\bar{\theta}^{2 \alpha}\left(\bar{X}_{2 \gamma}\right)\right)-\bar{X}_{2 \gamma}\left(\bar{\theta}^{2 \alpha}\left(\bar{X}_{2 \beta}\right)\right)-\bar{\theta}^{2 \alpha}\left(\left[\bar{X}_{2 \beta}, \bar{X}_{2 \gamma}\right]\right)=0 .
$$

Thus (1.8), (1.10) and (1.11) reduce to

$$
\begin{array}{ll}
\bar{\omega}_{1,3 \alpha}=\left(e^{-2 h} b_{3 \alpha, 1}^{3 \alpha}-2 e^{-2 h}\right) \theta^{3 \alpha}, & \bar{\omega}_{2 \alpha, 3 \beta}=\frac{1}{2} \sum_{\gamma} e^{-h} b_{2 \gamma, 2 \alpha}^{3 \beta} \theta^{2 \alpha}, \\
\bar{\omega}_{1,2 \alpha}=\left(e^{-h}+e^{-h} b_{2 \alpha, 1}^{2 \alpha}\right) \theta^{2 \alpha}, & \bar{\omega}_{2 \alpha, 2 \beta}=\bar{\omega}_{3 \alpha, 3 \beta}=0 .
\end{array}
$$

Thus the $\bar{\omega}_{\mu \nu}$ are exponentially decreasing, as are the $\bar{\Omega}_{\mu \nu}$. Notice that the decay is much faster than required.

The next proposition gives a slight generalization of the last calculation. The odd hypotheses highlight the special nature of the invariant metric.

Proposition 1.15. Let $M$ be a complete oriented even-dimensional manifold of finite topoloigcal type. Assume that in an open neighborhood of each component of the boundary, $T M=W_{2} \oplus \cdots \oplus W_{k} \oplus \mathbf{R}$ and the metric is of the form $f_{2}^{2}(h) g_{2} \oplus$ $\cdots \oplus f_{k}^{2}(h) g_{k} \oplus d h^{2}$ with $g_{i}$ a metric on $W_{i}$. If $\left[V_{i}, V_{i}\right]=0, f_{i}, f_{i}^{\prime} \rightarrow 0$ as $h \rightarrow \infty$ for $i=2, \ldots, k$, and $f_{i} / f_{2}$ and $\left(f_{i} / f_{2}\right)^{\prime}$ are bounded for $i=3, \ldots, k$, then $\chi(M)=$ $\int_{M} E(g)$.

PROOF. Under the hypotheses, (1.10) and (1.11) reduce to

$$
\begin{aligned}
& \bar{\omega}_{j \alpha, j \beta}=0, \\
& \bar{\omega}_{j \alpha, l \beta}=0, \quad \text { if } j, l \neq 2, \\
& \bar{\omega}_{2 \alpha, l \beta}=\frac{1}{2} \sum_{\gamma \neq \alpha} \frac{f_{l} f_{2}(1)}{f_{l}(1) f_{2}} b_{2 \alpha, 2 \gamma}^{l \beta} \theta^{2 \gamma} .
\end{aligned}
$$

As before, $\bar{\Omega}_{\mu \nu}$ is then bounded and $\bar{\omega}_{1, \rho}$ decays.

2. In this section we collect a few more classes of metrics which satisfy the Gauss-Bonnet Theorem.

The first example, which is well known, is the class of metrics which split as a product near $\partial M$ of a metric on $\partial M$ and a metric on $\mathbf{R}^{+}$. The metric need not be complete. We have $\omega_{1 \mu}=0$ for all $\mu$ by the uniqueness of solutions to the first structure equation. Thus $\Pi$ is identically zero.

We can also make an arbitrary metric constant near $\partial M$. That is, for a metric $g$ and fixed $h \in(1, \infty)$, set $g_{h}$ to be the metric which is $g$ on $M_{h-1 / 2}$ and such that $\left(g_{h}\right)_{i j}(x, k)=g_{i j}(x, h)$ for $k \geq h$ and $x \in \partial M$. Here the components are computed in coordinate charts of the form $V_{i} \times \mathbf{R}$, where the $V_{i}$ are charts for $\partial M$. On the gap between $\partial M_{h-1 / 2}$ and $\partial M_{h}$, the new metric is smoothed arbitrarily.

LEMMA 2.1. For $M$ even dimensional and of finite topological type,

$$
\chi(M)=\int_{M} E\left(g_{h}\right) \text {. }
$$


PrOOF. By construction, $g_{h}$ reflects smoothly across $\partial M_{k}, k>h$, to a metric $g_{h}^{\prime}$ on $M^{\prime}$, the double of $M_{k}$. The Gauss-Bonnet Theorem for the compact manifold $M^{\prime}$ gives

$$
\chi\left(M^{\prime}\right)=\int_{M} E\left(g_{h}^{\prime}\right)=2 \int_{M_{k}} E\left(g_{h}\right) .
$$

However, $\chi\left(M^{\prime}\right)=2 \chi\left(M_{k}\right)-\chi\left(\partial M_{k}\right)=2 \chi(M)$, since $\partial M_{k}$ is odd dimensional. Thus $\chi(M)=\int_{M_{k}} E\left(g_{h}\right)$, and letting $k$ go to infinity gives the result.

For these "cutoff" metrics, $\Pi$ need not vanish identically. For example, let $M$ be a surface diffeomorphic to $S^{2}$ minus a point. Choose coordinates $\left(x_{1}, x_{2}\right)$ in a collar of the boundary circle, with $x_{1}$ the circle coordinate. Finally, let $g$ be a cutoff metric on $M$. For surfaces, $\Pi=\omega_{12}$. Now $\theta^{2}=\sqrt{g_{22}} d x_{2}$ is a unit cotangent vector, and Gram-Schmidt gives $\theta^{1}=\left(g_{12} / \sqrt{g_{22}}\right) d x_{1}+\sqrt{g_{22}} d x_{2}$ as a unit cotangent vector orthogonal to $\theta^{2}$. Since $\partial g_{i j} / \partial x_{2}=0$ near the boundary, a quick calculation shows

$$
\omega_{12}=\frac{1}{2} \cdot \frac{1}{g_{22}} \cdot \frac{\partial g_{22}}{\partial x_{1}} d x_{1}
$$

near the boundary. Thus $\omega_{12}$ does not vanish in general, but as dictated by the lemma

$$
\int_{S^{1}} \omega_{12}=\frac{1}{2} \int_{S^{1}} d\left(\log g_{22}\right)=0
$$

For a surface $M$ of finite topological type, a complete metric in gradient flow coordinates is $f^{2} d \theta^{2}+d h^{2}$, where $\theta$ is the angular variable of the circular hypersurfaces of the Morse function. Again it is easy to check that $\omega_{12}=-(\partial f / \partial h) d \theta$. Since $\partial M_{h}$ is a union of circles $S_{h}^{1}, \ldots, S_{h}^{k}$, we see that Gauss-Bonnet is satisfied if and only if

$$
\sum_{i=1}^{k} \int_{S_{h}^{i}} \frac{\partial f}{\partial h} d \theta \rightarrow 0 \quad \text { as } h \rightarrow \infty .
$$

This formula is not the final word on Gauss-Bonnet for complete surfaces. Since the $h$ coordinate is gotten by integrating the gradient flow, putting a metric into gradient flow coordinates may be difficult.

As a final example avoiding gradient flow coordinates, we derive necessary and sufficient conditions for a surface of revolution in $\mathbf{R}^{3}$ to satisfy Gauss-Bonnet in the induced metric. Specifically, we consider the surface $M=\left\{z=f\left(x^{2}+y^{2}\right)\right\}$ where $f:(0, \infty) \rightarrow \mathbf{R}$ is $C^{\infty}$ and $f(0)=f^{\prime}(0)=0$. Thus $M$ is at least $C^{2}$ at $(0,0,0)$.

To write the metric $g$ on $M$ induced from $\mathbf{R}^{3}$, first note that on the piece of $M$ lying over $x^{2}+y^{2}=t^{2}$, the vector $(-y, x, 0)$ is tangent to $M$ and has length $t$. In other words, if $\theta$ is the usual angular variable, the component of $g$ in the horizontal direction is $t^{2} d \theta^{2}$. To find the other component, consider the curve $\left(t x, t y, f\left(t^{2}\right)\right)$ on $M$ orthogonal to the circles; here $x$ and $y$ are fixed and satisfy $x^{2}+y^{2}=1$. The tangent vector is $\left(x, y, 2 t f^{\prime}\left(t^{2}\right)\right)$ of length $\left(1+4 t^{2} f^{\prime}\left(t^{2}\right)^{2}\right)^{1 / 2}$. Thus in the $(\theta, t)$ coordinates covering $M-\{(0,0,0)\}$ the induced metric is

$$
t^{2} d \theta^{2}+\left(1+4 t^{2} f^{\prime}\left(t^{2}\right)^{2}\right) d t^{2}, \quad t \neq 0 .
$$

(If $f$ is concave, then $f$ serves as a Morse function on $M$. However, even in this case, finding gradient flow coordinates involves integrating $\left(1+4 t^{2} f^{\prime}\left(t^{2}\right)^{2}\right)^{-1} f^{\prime}\left(t^{2}\right)$.) 
Set $\alpha(t)=1+4 t^{2} f^{\prime}\left(t^{2}\right)^{2}$. If $M$ satisfies Gauss-Bonnet, then

$$
1=\chi(M)=\frac{1}{2 \pi} \int K d A=\frac{1}{2 \pi} \int_{0}^{2 \pi} \int_{0}^{\infty} K t \sqrt{\alpha} d t d \theta=\int_{0}^{\infty} K t \sqrt{\alpha} d t .
$$

A direct calculation of the Gaussian curvature $K$ from the metric (2.2) gives

$$
K=\alpha^{\prime} / 2 \alpha^{2} t, \quad t \neq 0
$$

Thus we want conditions on $f$ that ensure

$$
2=\int_{0}^{\infty} \alpha^{\prime} \alpha^{-3 / 2} d t
$$

But this is equivalent to $\alpha^{-1 / 2}(\infty)=0$, since $\lim _{\varepsilon \rightarrow 0} \alpha^{-1 / 2}(\varepsilon)=1$. Therefore, for such $M$, Gauss-Bonnet is equivalent to $t^{1 / 2} f^{\prime}(t) \rightarrow \pm \infty$ as $t \rightarrow \infty$.

PROPOSITION 2.4. Let $M$ be the surface of revolution $\left\{z=f\left(x^{2}+y^{2}\right)\right\}$ for $f:(0, \infty) \rightarrow \mathbf{R}$ with $f \in C^{\infty}$ and $f(0)=f^{\prime}(0)=0$. In the metric induced from $\mathbf{R}^{3}$,

$$
\chi(M)=\frac{1}{2 \pi} \int_{M} K d A \Leftrightarrow t^{1 / 2} f^{\prime}(t) \rightarrow \pm \infty \quad \text { as } t \rightarrow \infty .
$$

In particular, if $f$ is convex upwards or downwards (and nonconstant), GaussBonnet holds. Such $M$ are well known to have positive Gaussian curvature, as can be seen from (2.3) for $t \neq 0$. These surfaces clearly have infinite volume. For complete infinite volume surfaces in general, one knows only the Cohn-Vossen inequality $\chi(M) \geq(1 / 2 \pi) \int_{M} K d A$. Thus Proposition 2.4 somewhat complements Huber's result that finite volume surfaces satisfy Gauss-Bonnet, since by standard comparison results finite volume surfaces must be predominently negatively curved near infinity.

\section{REFERENCES}

[Bo] A. Borel, Introduction aux groupes arithmétiques, Hermann, Paris, 1969.

[CG] J. Cheeger and M. Gromov, On the characteristic numbers of complete manifolds of bounded curvature and finite volume, preprint.

[Ch] S.-S. Chern, On the curvatura integra on a Riemannian manifold, Ann. of Math. (2) 46 (1945), 674-684.

[Do] H. Donnelly, On the point spectrum for finite volume symmetric spaces of negative curvature, Comm. Partial Differential Equations 6 (1981), 963-992.

[Gr] M. Gromov, Volume and bounded cohomology, Inst. Hautes École Sci. Publ. Math. 56 (1982), 5-100.

[Ha] G. Harder, A Gauss-Bonnet formula for discrete arithmetically defined groups, Ann. Sci. École Norm. Sup. 4 (1971), 409-455.

[Hu] A. Huber, On subharmonic functions and differential geometry in the large, Comment. Math. Helv. 32 (1957), 12-72.

[Mi] J. Milnor, Morse theory, Princeton Univ. Press, Princeton, N.J., 1963.

[Ro] S. Rosenberg, Gauss-Bonnet theorems for noncompact surfaces, Proc. Amer. Math. Soc. 86 (1982), 184-185.

[Sp] E. Spanier, Algebraic topology, McGraw-Hill, New York, 1966.

Department of Mathematics, University of California, Berkeley, CaliforNIA 94720

Department of Mathematics, Brandeis University, Waltham, MassachuSETTS 02154 